\title{
PHOTOLUMINESCENCE STUDY OF POROUS SILICON AS PHOTOSENSITIZER OF SINGLET OXYGEN GENERATION
}

\author{
V.YU. TIMOSHENKO
}

PACS 78.60.-b, 78.67.-n

M.V. Lomonosov Moscow State University, Faculty of Physics

(C) 2011

(Leninskie Gory, 1, MSU, Moscow 119991, Russia; e-mail: timoshen@ physics.msu. ru)

Si nanocrystals with dimensions of about several nanometers in layers and powders of porous silicon (por-Si) act as photosensitizers of the generation of singlet oxygen which is a highly chemical reactive form of molecular oxygen. The photosensitized mechanism and the efficiency are investigated by means of the photoluminescence spectroscopy. The experimental data are discussed in view of possible biomedical applications of por-Si.

\section{Introduction}

It is known that singlet oxygen $\left({ }^{1} \mathrm{O}_{2}\right)$, which is an excited form of molecular oxygen $\left(\mathrm{O}_{2}\right)$, mediates important biochemical processes in living organisms [1]. Some examples of the biochemical reactivity of ${ }^{1} \mathrm{O}_{2}$ are lipid peroxidation, photohemolysis, and photodynamic therapy (PDT) combining drug and light treatment of tumors [2]. According to the quantum mechanics, the relaxation of singlet states back to the ground (triplet) state is "spinforbidden," and, thus, ${ }^{1} \mathrm{O}_{2}$ is characterized by a rather long lifetime [3], which is favorable for transferring the excess energy to another molecule. The transferred excess energy results in a sequence of oxidation reactions, which can be used for applications [4]. The procedure of ${ }^{1} \mathrm{O}_{2}$ generation, which is useful in biomedical applications and widely used, is a photosensitization including a certain substance called "photosensitizer", e.g., dye molecules [2]. Recently, it was found that Si nanocrystals (nc-Si) in porous silicon (por-Si) formed by the electrochemical method could be used to photosensitize the ${ }^{1} \mathrm{O}_{2}$ generation [5]. The observed efficient photosensitization was explained by the energy transfer from excitons in nc-Si to $\mathrm{O}_{2}$ molecules adsorbed on the nc-Si surface [6]. This process is favorable because of the highly developed surface $\left(10^{2} \div 10^{3} \mathrm{~m}^{2} / \mathrm{g}\right)$ of por-Si [7] and the long radiative lifetimes $\left(10^{-5} \div 10^{-3} \mathrm{~s}\right)$ of excitons confined in nc-Si [8].

The quenching of the exciton photoluminescence (PL) of por-Si with adsorbed $\mathrm{O}_{2}$ molecules was measured to clarify the energy transfer mechanism [6]. While the PL quenching is only an indirect evidence of the ${ }^{1} \mathrm{O}_{2}$ formation, the direct and quantitative data can be obtained by analyzing the radiative decay of ${ }^{1} \mathrm{O}_{2}$ molecules at $0.98 \mathrm{eV}$ $(1270 \mathrm{~nm})$ [4, 9-12]. In fact, the ${ }^{1} \mathrm{O}_{2}$ photosensitization by por-Si was quantified by means of the luminescence spectroscopy at $0.98 \mathrm{eV}[13]$. On the one hand, a lot of the experiments on the ${ }^{1} \mathrm{O}_{2}$ luminescence demonstrates the efficient generation of ${ }^{1} \mathrm{O}_{2}$ by the energy transfer from nc-Si [14]. On the other hand, the comparison of the ${ }^{1} \mathrm{O}_{2}$ luminescence intensity for por-Si and for the standard photosensitizers such as porphyrins indicated the strong deactivation (quenching) of ${ }^{1} \mathrm{O}_{2}$ molecules in por-Si structures [15], which limits significantly the net concentration of ${ }^{1} \mathrm{O}_{2}$ photosensitized by por-Si [16]. The electron paramagnetic resonance (EPR) technique can also provide information about the ${ }^{1} \mathrm{O}_{2}$ concentration in different reactions [4]. The X-band EPR spectroscopy of photoexcited por-Si in $\mathrm{O}_{2}$ atmosphere gave estimates of the concentration of photosensitized ${ }^{1} \mathrm{O}_{2}$ molecules to be about $10^{18} \div 10^{19} \mathrm{~cm}^{-3}$ under excitation with a photon flux of about $10^{19} \div 10^{20} \mathrm{~cm}^{-2}[17,18]$. Similar results were obtained by means of the Q-band EPR spectroscopy, which demonstrated a conversion of about $30 \%$ of $\mathrm{O}_{2}$ molecules in the singlet state upon the excitation of por-Si powder in oxygen atmosphere [19]. The ${ }^{1} \mathrm{O}_{2}$ photosensitization was observed for por-Si dispersed in various oxygen-saturated organic and inorganic liquids [13, 20, 21], as well in water [22]. Furthermore, the biomedical experiments in vitro demonstrated the suppression of the cancer cell proliferation by introducing photoexcited nc-Si, which was obtained from por-Si powder [23].

In the present paper, the basic properties of ${ }^{1} \mathrm{O}_{2}$ and the fundamental aspects of photosensitizing properties of nc-Si are analyzed by means of the PL spectroscopy. The experimental results are presented and discussed to make the quantitative information about the ${ }^{1} \mathrm{O}_{2}$ photosensitization and quenching. Besides the data on physical properties of por-Si, the results of biomedical experiments with por-Si are also discussed. 


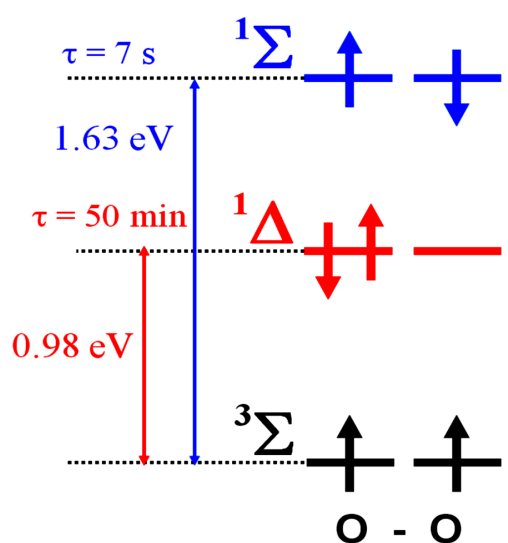

Fig. 1. Simplified diagram of the electronic states in a free $\mathrm{O}_{2}$ molecule. The radiative lifetimes and energies of the electronic transitions from the singlet states to the ground (triplet) one are indicated. Arrows mark the location and the orientation of the electron spins in $\mathrm{O}_{2}$ molecule

\section{Basic Properties of Singlet Oxygen}

Molecular oxygen is among the most important substances, because it is involved in a plenty of chemical and biochemical reactions in nature. It is known that the ground state of $\mathrm{O}_{2}$ is paramagnetic and characterized by the total spin equal to 1 . Molecular oxygen in the ground state is sometimes denoted as "triplet oxygen," and it is labeled as ${ }^{3} \mathrm{O}_{2}$ [4]. The singlet oxygen, which is labeled as ${ }^{1} \mathrm{O}_{2}$, has the total spin equal to 0 . The singlet oxygen was first observed in 1924 and then defined as the most reactive form of molecular oxygen (see, e.g., [24]). Several years later, the photosensitized generation of ${ }^{1} \mathrm{O}_{2}$ was discovered by $\mathrm{H}$. Kautsky [25].

According to quantum theory, the spin configuration of the ground energy state of $\mathrm{O}_{2}$ is characterized by unpaired electrons in two different molecular orbitals located at two different oxygen atoms, as it is schematically shown in Fig. 1. This spin configuration accounts for the paramagnetism of $\mathrm{O}_{2}$ in the ground triplet state, which is labeled as ${ }^{3} \Sigma$. The electronically excited states of $\mathrm{O}_{2}$ are singlet ones, and they are characterized by the energy excess of about $0.98 \mathrm{eV}\left({ }^{1} \Delta\right.$ state $)$ and $1.63 \mathrm{eV}$ $\left({ }^{1} \Sigma\right.$ state) in respect to the ground state (see Fig. 1$)$. The transition from ${ }^{3} \mathrm{O}_{2}$ to ${ }^{1} \mathrm{O}_{2}$ requires the spin-flip process, and the direct conversion of states via the absorption of photons is spin-forbidden in the first approximation. Since the photon absorption and emission are mirror-like processes, the radiative decay lifetimes of the ${ }^{1} \Delta$ and ${ }^{1} \Sigma$ states are extremely long: about $45 \mathrm{~min}$ and $7 \mathrm{~s}$, respectively [3]. Note that the observed lifetimes of the singlet states are considerably shorter in condensed phases

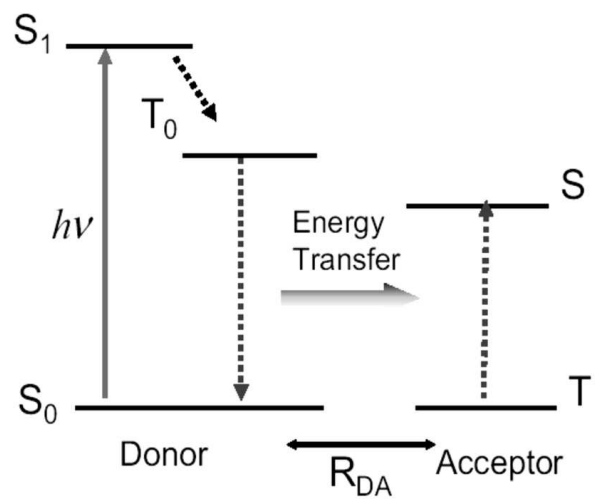

Fig. 2. A schematic presentation of the energy transfer process. The absorbed photon energy, $h \nu$, is marked by the solid vertical arrow. Block letters $\mathrm{S}$ and $\mathrm{T}$ denote singlet and triplet states, respectively. $R_{\mathrm{DA}}$ indicates the space separation between the donor and the acceptor

$\left(\sim 10^{-9} \mathrm{~s}\right.$ and $\sim 10^{-3} \mathrm{~s}$ for the ${ }^{1} \Sigma$ and ${ }^{1} \Delta$ states, respectively) because of collisions of ${ }^{1} \mathrm{O}_{2}$ with other molecules [1]. In addition, it was found that the radiative lifetime of the ${ }^{1} \Delta$ state decreases also down to $1-8 \mathrm{~s}$ in solvents such as benzene, chloroform, acetone, water, etc. [10].

The efficient method of the ${ }^{1} \mathrm{O}_{2}$ generation is based on the photosensitization process [2]. This process requires the presence of a light-absorbing substance, the photosensitizer (energy donor), which initiates the processes of energy transfer to a non-absorbing light substrate (energy acceptor), as shown schematically in Fig. 2. The efficient energy transfer from a donor to an acceptor must be downhill. Several types of excitation energy transfer mechanisms have been identified. The long-range process, which involves a "dipole-dipole" interaction between the excited singlet states of the donor and the acceptor, is called the Förster energy transfer [26]. The transfer efficiency depends on the spectral overlap of the donor emission band and the acceptor absorption band, the inverse-sixth power of the intermolecular separation, and the relative donor-acceptor orientation. A short-range mechanism, which is termed commonly as the Dexter mechanism [27], is based on the direct electron exchange between the donor and the acceptor.

The direct optical excitation is the evident but rather inefficient method of ${ }^{1} \mathrm{O}_{2}$ formation, because the optically excited electronic transitions between the ground ${ }^{3} \Sigma$ state and the singlet ones $\left({ }^{1} \Sigma\right.$ and $\left.{ }^{1} \Delta\right)$ are not allowed in the first approximation [2]. Nevertheless, it was demonstrated by Krasnovsky et al. that the direct excitation of ${ }^{1} \mathrm{O}_{2}$ in pure carbon tetrachloride via the transition ${ }^{3} \Sigma \rightarrow{ }^{1} \Delta$ could be realized under intense laser irradiation at a wavelength of about $1270 \mathrm{~nm}$. However, 
the corresponding absorption coefficient was 8 orders of magnitude less than that for the typical photosensitizer such as porphyrin [11].

The luminescence is the gold standard of the ${ }^{1} \mathrm{O}_{2}$ identification. As it was already mentioned, the direct optical excitation of $\mathrm{O}_{2}$ from the triplet state to the singlet one is not allowed in the first approximation. However, even for a free $\mathrm{O}_{2}$ molecule, the absorption lines of the electronic transitions from the ${ }^{3} \Sigma$ state to the ${ }^{1} \Delta$ and ${ }^{1} \Sigma$ states are observed at 1269 and $762 \mathrm{~nm}$, respectively, because of the wave function mixture and high-order processes [3]. At high oxygen pressures when oxygen-oxygen collisions are more probable or in liquid oxygen, the additional absorption bands are observed at 634 and $476 \mathrm{~nm}$. Energetically, these bands correspond to various combinations of $\mathrm{O}_{2}$ pairs of single molecule transitions, and, therefore, they can be assigned as simultaneous transitions in which a single photon is responsible for the excitation of two molecules [4].

The decay time of the ${ }^{1} \mathrm{O}_{2}$ luminescence in liquids, which is actually the lifetime, $\tau_{\Delta}$, of the ${ }^{1} \Delta$ state, is highly dependent on the medium, ranging from about $3 \mu \mathrm{s}$ in water to about $1 \mathrm{~ms}$ in $\mathrm{CF}_{3} \mathrm{Cl}$ [28] and up to 87 $\mathrm{ms}$ in $\mathrm{CCl}_{4}$ [10]. High values of $\tau_{\Delta}$ about $25-100 \mathrm{~ms}$ were also observed in other solvents whose molecules did not have hydrogen atoms such as $\mathrm{C}_{6} \mathrm{~F}_{6}$ [29]. An essentially unambiguous test for ${ }^{1} \mathrm{O}_{2}$ in aqueous systems is based on increasing the lifetime $\tau_{\Delta}$ of the phosphorescence from $3 \mu \mathrm{s}$ in $\mathrm{H}_{2} \mathrm{O}$ to $30 \mu \mathrm{s}$ in $\mathrm{D}_{2} \mathrm{O}[9,10]$. According to the recent results of Snydler et al., $\tau_{\Delta}$ in $\mathrm{D}_{2} \mathrm{O}$ approaches 68 $\mu \mathrm{s}$ [30]. Despite a significant increase of the lifetime in perdeuterated solvents, the rates of reactions mediated by ${ }^{1} \mathrm{O}_{2}$ are accelerated usually less than 10 -fold owing to competing quenching reactions for ${ }^{1} \mathrm{O}_{2}[11,12]$. There is an approximate correlation between the solvent-induced decay lifetime of ${ }^{1} \mathrm{O}_{2}$ and the infrared absorption of the medium near $1270 \mathrm{~nm}$, suggesting that ${ }^{1} \mathrm{O}_{2}$ decays, by transferring its electronic excitation energy to the vibrational energy of the solvent.

\section{Photosensitized Generation of Singlet Oxygen by Si Nanocrystals}

\subsection{Basic properties of the electronic excitation in por-Si}

In order to clarify the mechanisms of photosensitized generation of ${ }^{1} \mathrm{O}_{2}$ by por-Si, let us firstly analyze the basic properties of the electronic excitation in this material. Many years ago, por-Si was invented and investigated as a new morphological form of silicon formed by the electrochemical etching of bulk crystalline silicon (c$\mathrm{Si}$ ) wafers in hydrofluoric acid solutions [31]. Now it is known that, for substrates of lightly doped p-type c-Si wafers, the formed por-Si is microporous, i.e. the mean pore size is smaller than $2 \mathrm{~nm}$ [32]. For high pore densities, which can be achieved at a certain etching regime, microporous por-Si consists of interconnected nc-Si with average sizes varying from 1 to $5 \mathrm{~nm}$, which depends on the preparation conditions [7]. The quantum confinement for charge carriers in such small nc-Si results in an increase of the energy gap [33]. The confinement effect is found to be strongest for spherical clusters of $\mathrm{Si}$ atoms, i.e. for smallest nc-Si with size restrictions in three dimensions [34].

Due to the quantum confinement and the size distribution of nc-Si, the effective band-gap energy of microporous Si ranges typically from $1.2 \mathrm{eV}$ up to $2.5 \mathrm{eV}$, as it was theoretically shown in [35]. Experimental data of the absorption coefficient of mesoporous por-Si demonstrate that, for the photon energy above $3 \mathrm{eV}$, the main contribution to the absorption is due to the transitions above the direct gap of $\mathrm{c}-\mathrm{Si}$, and then the spectrum is similar to that of c-Si [8]. In this spectral range, the absorption coefficient can be well described in the effective medium approximation [36]. The shift of the absorption curve to higher energies is due to the reduced density and the quantum confinement in nc-Si. Only at high energies when all crystallites are absorbing in the range of a high density of states, this discrepancy becomes smaller and, finally, vanishes at the energy of the direct transitions [8]. Note that the absorption coefficient of por-Si shows the continuous spectral dependence, which is different from that for dyes [2]. This fact is important for the photosensitizing properties of por-Si, which can be realized under excitation in the broad spectral range above $1.4 \mathrm{eV}[14]$.

While the photoluminescence (PL) of por-Si is sensitive to details of the electrochemical formation and the storage, there are unambiguous evidences of the quantum confinement effect on the PL band position [32]. For example, it is possible to tune the PL band position of por-Si in the spectral range from 1.1 to $2.5 \mathrm{eV}$ by using different c-Si substrates and etching conditions [8]. The PL properties of por-Si are also dependent on defects and contaminations, which can take place on the highly developed surface of nc-Si [32]. The influence of defect states on the optical and electronic properties of por-Si was confirmed by many experimental methods such as the optical absorption spectroscopy [32, 38], photoacoustics [39, 40], and EPR [32, 42]. 


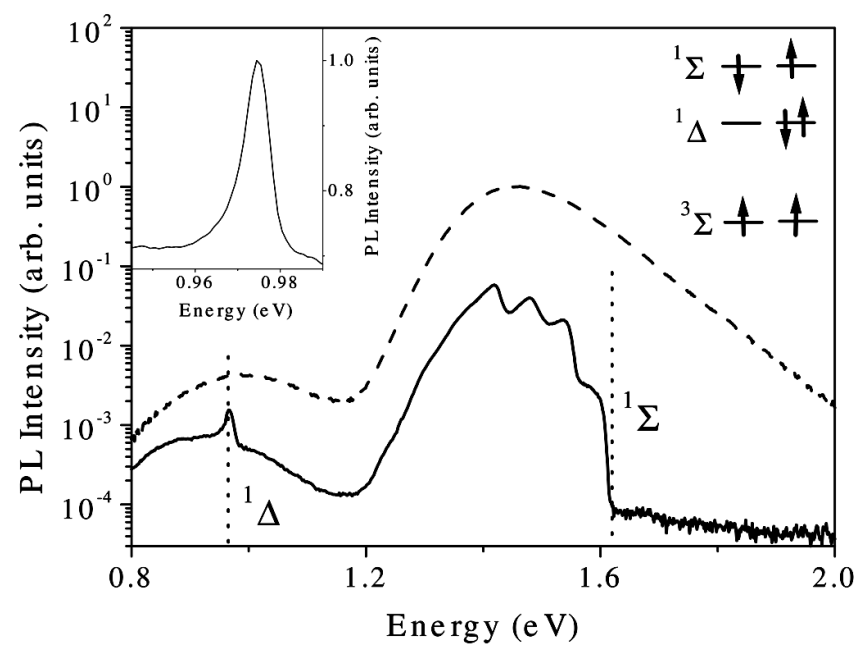

Fig. 3. PL spectra of por-Si in vacuum (dashed line) and in the presence of adsorbed oxygen molecules (solid line). Transition energies from the excited states $\left({ }^{1} \Delta,{ }^{1} \Sigma\right)$ of ${ }^{1} \mathrm{O}_{2}$ to the ground state are indicated by dashed vertical lines. Spin configurations and spectroscopic labeling of molecular oxygen states are shown. Inset depicts the PL line related to the ${ }^{1} \Delta \rightarrow{ }^{1} \Sigma$ transition in $\mathrm{O}_{2}$. Temperature $T=5 \mathrm{~K}$, excitation photon energy $E_{\text {exc }}=2.41 \mathrm{eV}$ [6]

The Coulomb interaction between photoexcited electrons and holes in nc-Si influences strongly their energies and then the PL photon energy [35]. The attractive Coloumb interaction for charge carriers in nc-Si results in the formation of excitons with relatively large binding energies of about hundreds of $\mathrm{meV}$, which determines possibilities for the excitons to be stable at room temperature [33, 35]. Furthermore, the exchange interaction in an exciton can remove the spin degeneracy for the exciton states. It results in two possible spin configurations for the exciton in nc-Si, which consist of optically active spin-singlet states and optically inactive spin-triplet ones. The triplet exciton state is lowered in energy in respect to the singlet state because of the electron-hole exchange interaction [37]. Despite a small singlet-triplet splitting being in the range from 1 to $30 \mathrm{meV}$, the main part of excitons $(75 \%)$ reside in the threefold degenerated triplet state even at elevated temperatures [8].

The long lifetime of the excitons in nc-Si is governed by the indirect nature of the optical transition and the corresponding spin selection rules. At cryogenic temperatures, only the triplet exciton state is occupied, and the radiative decay proceeds on a millisecond time scale. At room temperature, the excitons persist in the triplet state on a time scale comparable to the radiative lifetime of singlet excitons, being 10-100 $\mu$ s [8]. Thus, the electronic structure of excitons in nc-Si is very similar to that of dye molecules. The morphology of por-Si results in a huge accessible internal surface area up to $\sim 1000$ $\mathrm{m}^{2} / \mathrm{cm}^{3}$ [8], which permits a direct contact between the whole nc-Si assembly and molecules present in the interconnected pores. Therefore, por-Si meets the main requests on a photosensitizer of the ${ }^{1} \mathrm{O}_{2}$ generation.

\subsection{Photosensitizing properties of por-Si at cryogenic temperatures}

A strong interaction of oxygen molecules with photoexcited nc-Si in por-Si was clearly observed at cryogenic temperatures $[5,6]$. The investigated samples of por-Si were prepared by the standard electrochemical etching of (100)-oriented boron-doped c-Si wafers with a resistivity of $10-20 \Omega \cdot \mathrm{cm}$ in a solution of hydrofluoric acid. The current density and the etching time were $50 \mathrm{~mA} / \mathrm{cm}^{2}$ and $1 \mathrm{~h}$, respectively. The prepared free standing por-Si layers were dried in air for several hours. Then the prepared layers were milled to get a powder assembled from nc-Si and their agglomerates. The powder was kept in a vacuum chamber in oil-free vacuum with a residual pressure of about $10^{-5}$ Torr. The exciton PL spectrum of por-Si measured in vacuum at low temperatures is characterized by a broad featureless emission band located in the visible region (dashed line in Fig. 3). The spectral position and the bandwidth reflect the wide band-gap distribution of the nc-Si assembly. Intrinsic defects commonly attributed to Si dangling bonds are responsible for the infrared PL band below $1 \mathrm{eV}$ [7]. The adsorption of $\mathrm{O}_{2}$ on the nc-Si surface resulted in a strong suppression of both the exciton PL and the defect PL band (solid line in Fig. 3). The PL quenching by $\mathrm{O}_{2}$ adsorption is maximal for the PL photon energies above $1.63 \mathrm{eV}$. The latter value corresponds to the ${ }^{3} \Sigma \rightarrow{ }^{1} \Sigma$ transition energy in an $\mathrm{O}_{2}$ molecule. At the same time, the PL line at $0.98 \mathrm{eV}$, which is related to the radiative transition ${ }^{1} \Delta \rightarrow{ }^{1} \Sigma$, appeared after the oxygen adsorption in por$\mathrm{Si}$ (see inset in Fig. 3). These spectroscopic data give direct evidences of the ${ }^{1} \mathrm{O}_{2}$ photosensitization by por-Si.

A detailed description of the energy-transfer mechanism responsible for the photosensitized generation of ${ }^{1} \mathrm{O}_{2}$ can be obtained from the spectral dependence of the PL quenching strength, which can be defined as follows [6]:

$Q=I_{\mathrm{PL}}^{\mathrm{vac}} / I_{\mathrm{PL}}^{\mathrm{ox}}$

Here, $I_{\mathrm{PL}}^{\mathrm{vac}}$ and $I_{\mathrm{PL}}^{\mathrm{ox}}$ are the PL intensities of por-Si in vacuum and in oxygen ambient, respectively. The efficiency of the energy transfer from excitons in nc-Si to 
$\mathrm{O}_{2}$ molecules can be obviously calculated by using the following expression:

$\eta=1-Q^{-1}$

The spectra of $Q\left(h \nu_{\mathrm{PL}}\right)$ and $\eta\left(h \nu_{\mathrm{PL}}\right)$ demonstrate both the main maximum at $1.63 \mathrm{eV}(Q \gg 1, \eta \sim 1)$ and an additional maximum at $h \nu_{\mathrm{PL}}$ of about $57 \mathrm{meV}$ below the ${ }^{1} \Sigma$ state energy, as well as numerous maxima separated by energy of about $63 \mathrm{meV}[6]$. These maxima correspond to the energy transfer from nc-Si with band gaps, which do not match resonantly the excitation energies of the singlet states of $\mathrm{O}_{2}$. A local maximum separated by $57 \mathrm{meV}$ from the main maximum at $1.63 \mathrm{eV}$ is an evidence of the indirect radiative transition in nc$\mathrm{Si}$, where the participation of the momentum-conserving TO phonon with an energy of $57 \mathrm{eV}$ is required [8]. The excess of the exciton energy with respect to the energies of the ${ }^{1} \Delta$ and ${ }^{1} \Sigma$ states is released by the emission of TO phonons with an energy of $63 \mathrm{eV}[6]$.

The dynamics of energy transfer can be deduced from the PL transients of por-Si in the presence of adsorbed $\mathrm{O}_{2}$ molecules. The energy transfer time from one $\mathrm{Si}$ nanocrystal to a single $\mathrm{O}_{2}$ molecule is not accessible experimentally, since a large number of nc-Si contributes to the PL at a certain emission energy. At low temperatures in vacuum, the $\mathrm{PL}$ decay time, $\tau_{\mathrm{PL}}$, is approximately equal to the radiative lifetime of excitons in nc-Si, $\tau_{\mathrm{ex}}$, and it is about $\sim 10^{-3} \mathrm{~s}$ independently on the PL photon energy [8]. The PL quenching by the $\mathrm{O}_{2}$ adsorption is accompanied by a significant decrease of the PL decay time, because the adsorbed $\mathrm{O}_{2}$ molecules on the nc-Si surface represent a channel for the nonradiative recombination of excitons. The spectral dependence of $\tau_{\mathrm{PL}}$ follows the quenching strength spectrum. The shortest $\tau_{\mathrm{PL}}$ is observed for the nanocrystals, which transfer their excitation most efficiently $[5,6]$. Increasing the concentration of adsorbed $\mathrm{O}_{2}$ molecules results in a stronger PL quenching and the further decrease of $\tau_{\mathrm{PL}}$ [7]. The timeresolved PL studies performed with nanosecond time resolution confirmed this spectral behavior of the exciton lifetimes of por-Si in oxygen ambient [22].

The average time of the energy transfer from excitons in the nc-Si assembly to $\mathrm{O}_{2}$ molecules can be calculated from the expression

$\tau_{\mathrm{ET}}^{-1}=\tau_{\mathrm{PL}}^{-1}-\tau_{\mathrm{ex}}^{-1}$

In the regime of the strongest quenching, the values of $\tau_{\mathrm{ET}}$ for the ${ }^{1} \Delta$ and ${ }^{1} \Sigma$ states are $50-100 \mu \mathrm{s}$ and $<3 \mu \mathrm{s}$, respectively $[6]$.
Basically, the dipole-dipole interaction [26] or direct electron exchange [27] coupling can account for the energy transfer from excitons to $\mathrm{O}_{2}$. The long-range Coulomb interaction is efficient, when it is based on optically allowed transitions of the donor and the acceptor. Therefore, it cannot be probably applied to the triplettriplet annihilation of excitons and $\mathrm{O}_{2}$ followed by the ${ }^{1} \mathrm{O}_{2}$ creation. However, for the electron exchange mechanism, these spin restrictions are lifted, and the triplet exciton annihilation accompanied by the spin-flip excitation of an $\mathrm{O}_{2}$ molecule is the allowed process. In this case, the energy transfer rate is defined by the spatial overlap of the electronic wave functions of the interacting species and depends exponentially on the donor-acceptor distance [27]. The latter can be varied by a modification of the nc-Si surface. Indeed, as-prepared por-Si exhibits a hydrogen-terminated surface with characteristic absorption lines of the vibrational modes of $\mathrm{Si}-\mathrm{H}$ bonds [31]. The annealing of the porous layers at $200{ }^{\circ} \mathrm{C}$ in oxygen ambient introduces a monolayer of back-bonded oxygen on the nc-Si surface, while the hydrogen passivation of the surface is preserved. For oxidized nc-Si, the increased spacing between confined excitons and adsorbed $\mathrm{O}_{2}$ molecules is on the order of double the length of the $\mathrm{Si}-\mathrm{O}$ bond $(\sim 3 \AA)$. This critically affects the efficiency of the electron exchange interaction. Contrary to a strong coupling for hydrogen-terminated nanocrystals, the PL quenching efficiency is reduced by orders of magnitude, if a thin oxide barrier is present [5].

Besides the quenched exciton PL in por-Si with adsorbed $\mathrm{O}_{2}$ molecules, there are infrared PL bands related to defects in nc-Si and radiative transitions in the photosensitized ${ }^{1} \mathrm{O}_{2}$ (see Fig. 3). The relevant time scale of the infrared PL was determined from time-resolved measurements. Inset in Fig. 4 shows the transients of the defect-related emission for por-Si in vacuum detected at photon energies of 0.953 and $0.973 \mathrm{eV}$. The rise time coincides within the response time of the used experimental setup ( $100 \mathrm{~ns})$ and yields the upper limit for the capture time of carriers to defect states [6]. The nonexponential decay proceeds on a microsecond time scale and does not vary with the PL photon energy. In the presence of adsorbed $\mathrm{O}_{2}$ molecules, the radiative decay of the ${ }^{1} \Delta$ state of ${ }^{1} \mathrm{O}_{2}$ is observed at a detection energy of $0.953 \mathrm{eV}$. The initial fast decay is attributed to the defect-related PL background. The slow component with the lifetime $\tau_{\Delta} \approx 0.5 \mathrm{~ms}$ accounts for the ${ }^{1} \Delta \rightarrow{ }^{3} \Sigma$ transition in $\mathrm{O}_{2}$. This lifetime is significantly shorter that the radiative lifetime of a free $\mathrm{O}_{2}$ molecule (see Section 2). The shortening of $\tau_{\Delta}$ can be explained by the interaction of the ${ }^{1} \Delta$ state of ${ }^{1} \mathrm{O}_{2}$ with nc-Si. The same 


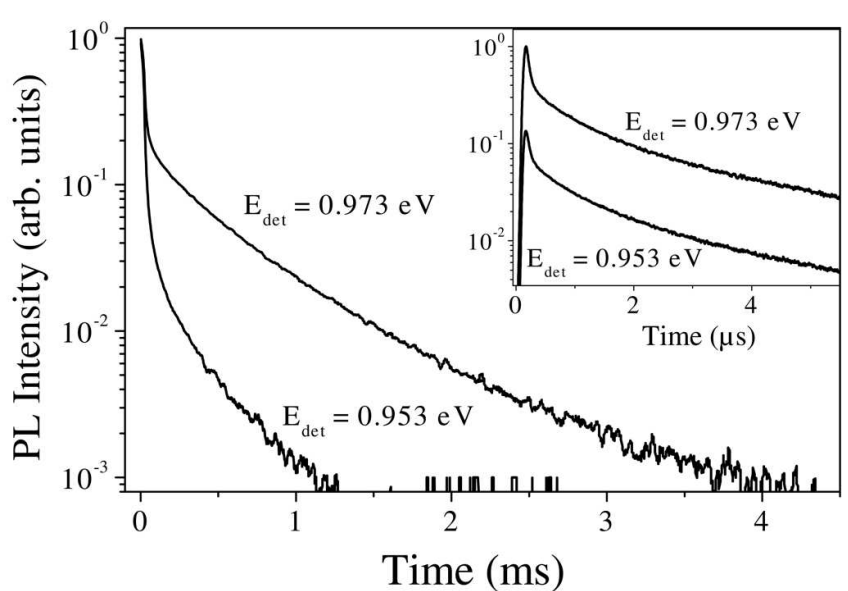

Fig. 4. Transients of the infrared $\mathrm{PL}$ of ${ }^{1} \mathrm{O}_{2}$ (detection energy $\left.E_{\text {det }}=0.973 \mathrm{eV}\right)$ and the defect-related PL at $h \nu_{\mathrm{PL}}=0.953 \mathrm{eV}$ of por-Si with adsorbed $\mathrm{O}_{2}$ molecules at $T=5 \mathrm{~K}$. Inset: $\mathrm{PL}$ transients of the defect-related PL of por-Si in vacuum [6]

interaction should also influence the radiative lifetime $\tau_{r}$ of adsorbed ${ }^{1} \mathrm{O}_{2}$ molecules. First, the quantum yield $\eta_{r} \approx 7 \times 10^{-5}$ of the radiative decay of the ${ }^{1} \Delta$ state is estimated from the integral intensity of the exciton PL band. Then $\tau_{r} \approx 7 \mathrm{~s}$ is obtained by using the simple relation $\tau_{r}=\tau_{\Delta} \eta_{r}$. On the one hand, the obtained $\tau_{r}$ is much shorter than the undisturbed radiative lifetime of about $50 \mathrm{~min}$ [3]. On the other hand, $\tau_{r} \approx 7 \mathrm{~s}$ is close to the radiative lifetimes of ${ }^{1} \mathrm{O}_{2}$ in different solvents [12]. Therefore, por-Si acts as both the photosensitizer and a quencher of ${ }^{1} \mathrm{O}_{2}$.

\subsection{Photosensitizing properties of por-Si at room temperature}

While the above-discussed PL experiments at cryogenic temperatures are important to clarify details of the photosensitization of ${ }^{1} \mathrm{O}_{2}$ by por-Si, the ${ }^{1} \mathrm{O}_{2}$ generation at room temperature is of most practical interest. Contrary to cryogenic temperatures, the optimal interaction conditions for nc-Si $/ \mathrm{O}_{2}$ system are not fulfilled at elevated temperatures. Because of the thermally activated desorption, a small spatial separation is realized only during a short time of collisions between $\mathrm{O}_{2}$ molecules and the nc-Si surface. Additionally, the exciton lifetime and the occupation number of the spin-triplet state of the exciton decrease with rising temperature [36]. This results in a weaker PL quenching, which scales with the collision rate, i.e., the gas pressure [6], and in a broad spectral resonance of the energy transfer to the ${ }^{1} \sum$ state as it is shown in Fig. 5. The corresponding spectrum of $\eta$ is presented in the inset in Fig. 5. On the one hand,

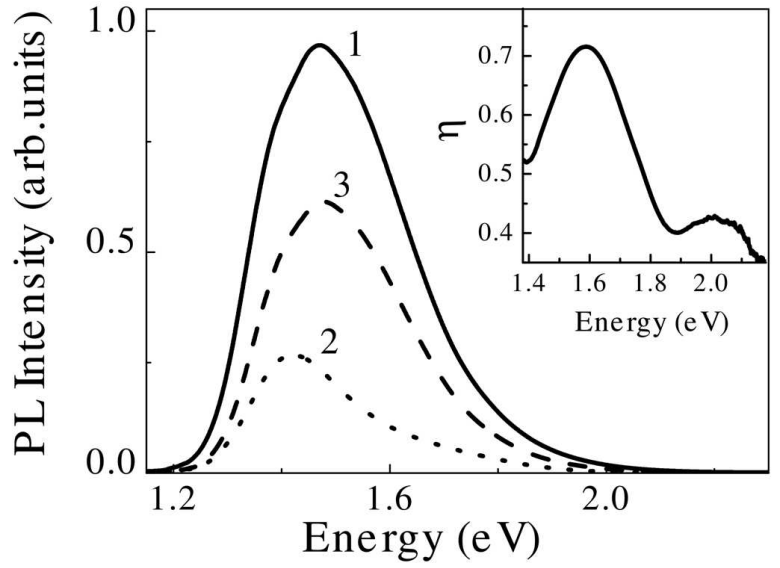

Fig. 5. Spectra of the room-temperature PL of por-Si in vacuum (1), in oxygen ambient at $p=760$ Torr (2), and just after evacuation (3). Inset shows the spectral dependence of the efficiency of energy transfer

the maximal value of $\eta \approx 0.7$, which occurs at $1.63 \mathrm{eV}$, indicates the rather efficient energy transfer from excitons in nc-Si with resonant energies to $\mathrm{O}_{2}$ molecules. On the other hand, the spectrally averaged efficiency of the energy transfer for por-Si, which can be obtained by the simple integration of $\eta\left(h \nu_{\mathrm{PL}}\right)$, does not exceed 0.5. Taking into account that the quantum efficiency of the exciton PL is typically smaller $10 \%$, one can estimate the quantum efficiency of the ${ }^{1} \mathrm{O}_{2}$ generation at room temperature to be lower $5 \%$.

The dependence of the ${ }^{1} \mathrm{O}_{2}$ generation efficiency on the $\mathrm{O}_{2}$ ambient pressure at room temperature is well described within the Langmuir approach for the molecule adsorption [43]. Figure 6 shows the value of $\eta$ measured at $1.63 \mathrm{eV}$ for a por-Si powder in the molecular oxygen atmosphere as a function of the $\mathrm{O}_{2}$ pressure. The experimental data are fitted by the expression

$\eta=\frac{\eta_{0}}{1+p_{0} / p}$

where $\eta_{0}$ is the energy transfer efficiency, which corresponds to one monolayer of adsorbed oxygen molecules, $p$ is the oxygen pressure, $p_{0}$ is the pressure, which corresponds to a half monolayer coverage of the nc-Si surface. The best fit of the experimental data in Fig. 6 gives the parameter $p_{0}=200$ Torr. In the entire pressure range, a decrease of the temperature increases the mean number of adsorbed molecules and enhances the efficiency of energy transfer [6, 41]. The steady state concentration of ${ }^{1} \mathrm{O}_{2}$ is a product of its generation rate and the collision-related deactivation rate. Because both quantities are rising with the oxygen ambient pressure [4], 


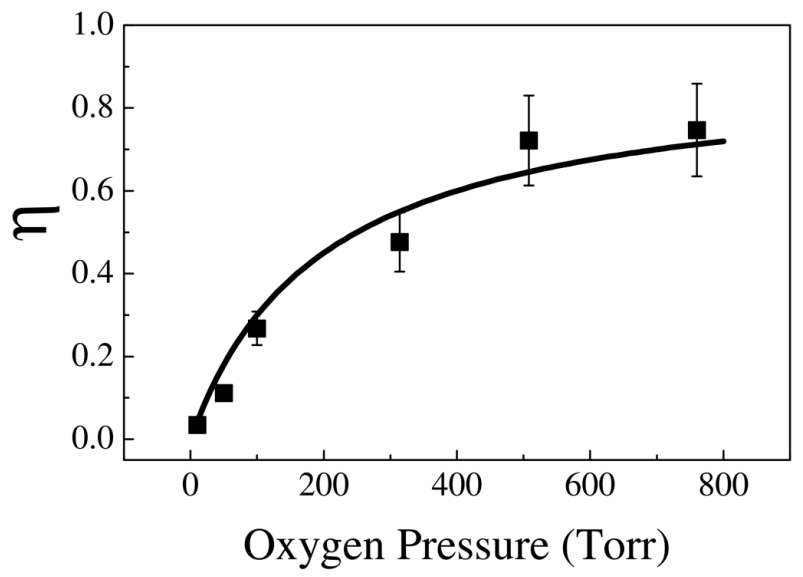

Fig. 6. Dependence of the energy transfer efficiency on the oxygen pressure for por-Si at room temperature. The experimental data (squares) are calculated by using Eqs. (1) and (2) from the PL intensities at $1.63 \mathrm{eV}$ [42]. The fit (solid line) is obtained by using Eq. (4)

there is the optimal $p_{o}$ to achieve the maximal steady state concentration of ${ }^{1} \mathrm{O}_{2}$ [6]. The good agreement between the experimental data and the Langmuir model indicates that the amount of photosensitized singlet oxygen is almost proportional of the number of adsorbed oxygen molecules. This fact has to be taken into account for different applications of the photosensitizing properties of por-Si.

Besides the characteristic energy of about $1.63 \mathrm{eV}$ (the ${ }^{1} \Sigma \rightarrow{ }^{3} \Sigma$, transition energy in $\mathrm{O}_{2}$ molecules), the spectrally selective quenching of the PL intensity of por-Si can demonstrate an additional maximum at a photon energy of about $2-2.2 \mathrm{eV}$ (see the inset in Fig. 5). The high-energy quenching maximum is usually observed for por-Si samples with a broad distribution of nc-Si sizes and surface conditions, e.g. for por-Si powders obtained by the mechanical grinding of por-Si films or by the stain etching of a polycrystalline Si powder [14]. The origin of the high energy quenching can be related to the formation of superoxide radicals, i.e. $\mathrm{O}_{2}^{-}$[6]. There are no unambiguous evidences of this characteristic energy of the $\mathrm{O}_{2}^{-}$formation. But the latter process is known to be more energy consumed than the ${ }^{1} \mathrm{O}_{2}$ generation (see Refs. [3, 4]). The PL quenching at $2-2.2 \mathrm{eV}$ can be also explained by the charging of nc-Si due to the $\mathrm{O}_{2}^{-}$formation on the nc-Si surface. The positively charged volume of nc-Si results in an increase of the nonradiative Auger recombination [6].

Additional information about the ${ }^{1} \mathrm{O}_{2}$ photosensitization at room temperature is obtained from the PL transient measurements. Figure 7 presents several PL tran-

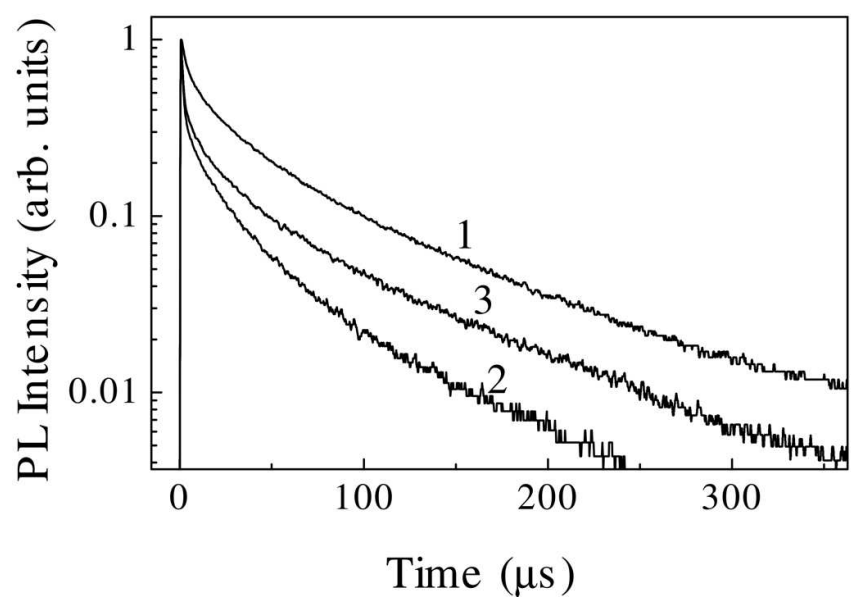

Fig. 7. Transients of the room-temperature PL at $1.63 \mathrm{eV}$ of por-Si in vacuum (1), in oxygen ambient at $p=760$ Torr (2) and just after evacuation (3)

sients of por-Si in gaseous oxygen ambient [40]. The PL transients are not actually monoexponential. However, for longer times (typically, $>50 \mu$ s after the excitation laser pulse), they can be well fitted by the monoexponential function as follows:

$I_{\mathrm{PL}}(t)=I_{\mathrm{PL}}(0) \exp \left(-t / \tau_{\mathrm{PL}}\right)$,

where $\tau_{\mathrm{PL}}$ is the PL lifetime, and $I_{\mathrm{PL}}(0)$ is the PL intensity just after the excitation. The values of $\tau_{\mathrm{PL}}$ for the samples in vacuum are found to be within a microsecond time interval, which is in good agreement with the singlet lifetimes of excitons in nc-Si $[8,34]$.

The admission of $\mathrm{O}_{2}$ causes a decrease of $\tau_{\mathrm{PL}}$ (see Fig. 7), and this effect is stronger at higher oxygen pressures (see Fig. 8). The strongest lifetime shortening occurs at a PL energy of $1.6 \mathrm{eV}$, which correlates with the spectral maximum of the PL intensity quenching. The PL lifetime shortening after the oxygen adsorption was nearly reversible at low oxygen pressures [41]. The energy transfer time can be obtained from the PL decay times by applying Eq. (3), where $\tau_{\text {ex }}$ has to be substituted by $\tau_{\mathrm{PL}}$ in vacuum. Figure 8 shows that $\tau_{\mathrm{PL}}$ decreases strongly with increasing the pressure of oxygen, while $\tau_{\mathrm{PL}}$ in vacuum does not sensitive to previous admissions of oxygen. These data indicate that the singlet oxygen-induced defect formation results mainly in a decrease of the number of luminescent nc-Si. This can be understood taking into account the short lifetime $(<1 \mu \mathrm{s})$ of the nonradiative recombination of photoexcited charge carriers in nc-Si with dangling bonds [32].

At room temperature, the infrared PL of por-Si in oxygen ambient can also give important information about 


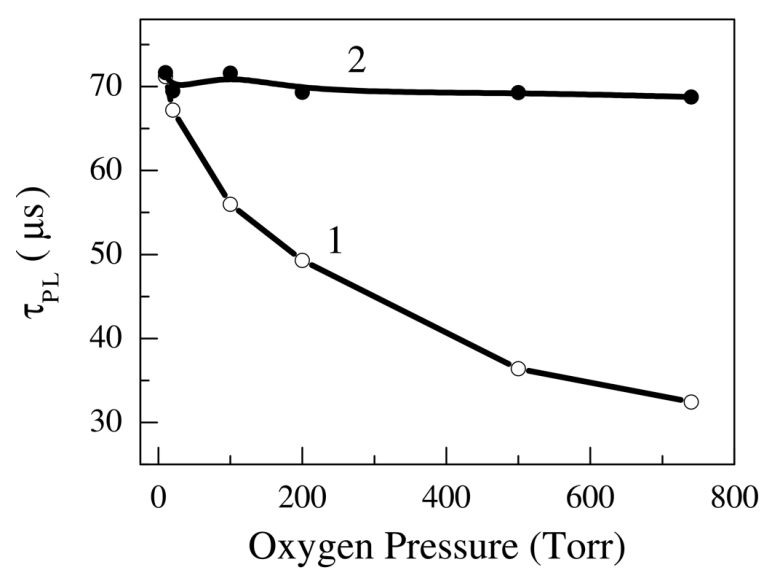

Fig. 8. Dependences of the PL lifetime, which is estimated from the PL transients by fitting with Eq. (5), of por-Si in oxygen atmosphere (1) and in vacuum just after the evacuation (2) vs the oxygen pressure. The PL photon energy was $1.63 \mathrm{eV} . T=300 \mathrm{~K}$

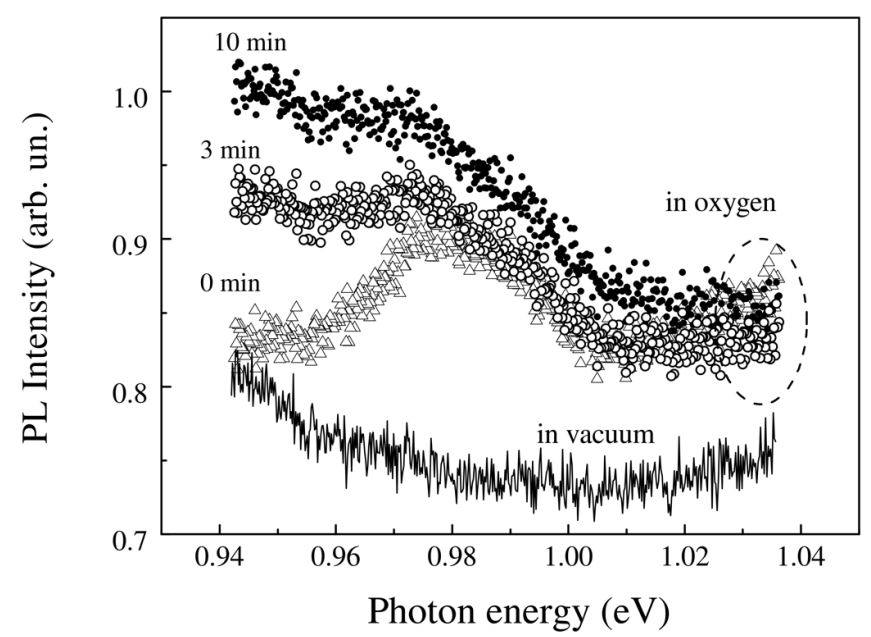

Fig. 9. PL spectra of por-Si measured in vacuum (lowest curve) and in oxygen atmosphere at $p=760$ Torr for different times after the illumination (indicated near the corresponding curves). Accumulation time of the PL signal was $30 \mathrm{~s}$. $E_{\text {exc }}=2.33 \mathrm{eV}$, $I_{\text {exc }}=0.1 \mathrm{~W} / \mathrm{cm}^{2}, T=300 \mathrm{~K}[16]$

the photosensitized generation of ${ }^{1} \mathrm{O}_{2}$, as it was demonstrated above at low temperatures (see Section 3.2). Since the radiative transitions in ${ }^{1} \mathrm{O}_{2}$ molecules are characterized by an extremely low probability, the experimental observation of the ${ }^{1} \mathrm{O}_{2}$ phosphorescence at $0.98 \mathrm{eV}$ requires a very sensitive detection system and is possible only for por-Si with rather efficient both the exciton PL and the generation of ${ }^{1} \mathrm{O}_{2}$. It can be realized for highly porous samples of por-Si with well-passivated surfaces of nc-Si [16]. Figure 9 shows the infrared PL spectra of such por-Si in oxygen atmosphere. The PL spectrum

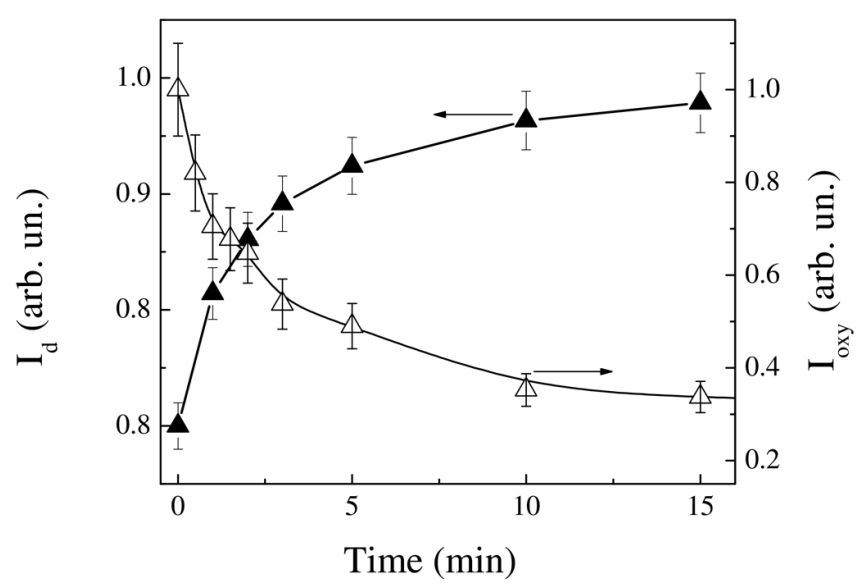

Fig. 10. Time-dependent intensities of the defect-related PL at 0.94-0.95 eV $\left(I_{d}\right.$, black triangles $)$ and the ${ }^{1} \mathrm{O}_{2}$ luminescence at $0.98 \mathrm{eV}$ ( $I_{\text {oxy }}$, open triangles) under excitation at a photon energy of $2.33 \mathrm{eV}$ and an intensity of $0.1 \mathrm{~W} / \mathrm{cm}^{2}$ at $T=300 \mathrm{~K}$

for por-Si in vacuum shows a featureless emission band related to defect states such as Si dangling bonds (DBs) in the band gap of nc-Si (see, e.g., [7, 32]). The PL line of the ${ }^{1} \mathrm{O}_{2}$ phosphorescence at $0.98 \mathrm{eV}$ appears just after the admission of $\mathrm{O}_{2}$ to por-Si. For several minutes after the $\mathrm{O}_{2}$ admission, the ${ }^{1} \mathrm{O}_{2}$ phosphorescence intensity decreases, while the intensity of the defect-related band increases, as it is shown in Fig. 9. The relative intensity of the ${ }^{1} \mathrm{O}_{2}$ phosphorescence intensity was found to decrease in the por-Si samples subjected to the mechanical grinding, which resulted in increasing the PL intensity of the defect-related band [16].

Figure 10 shows the temporal dependences of the intensities of ${ }^{1} \mathrm{O}_{2}$ phosphorescence, $I_{\text {oxy }}$, at $0.98 \mathrm{eV}$ and defect-related PL, $I_{\mathrm{d}}$, at $0.94-0.95 \mathrm{eV}$ for por-Si photoexcitated at room temperature. The obtained results indicate the formation of new defect states on nc-Si surfaces due to the photosensitized generation of ${ }^{1} \mathrm{O}_{2}[16]$. The most probable reason for the defect formation is the photo-induced oxidation of nc-Si, which is stimulated by ${ }^{1} \mathrm{O}_{2}$ and accompanied by the irreversible quenching of the exciton PL [43]. The latter process induces decreasing the ${ }^{1} \mathrm{O}_{2}$ photosensitization efficiency. Then the ${ }^{1} \mathrm{O}_{2}$ phosphorescence intensity drops down (see Fig. 10). Therefore, por-Si acts as both the photosensitizer and the quencher of ${ }^{1} \mathrm{O}_{2}$. From the comparison of yields of the ${ }^{1} \mathrm{O}_{2}$ phosphorescence and the exciton emission of por-Si, the ${ }^{1} \mathrm{O}_{2}$ lifetime $\tau_{\Delta} \approx 15 \mathrm{~ms}$ was estimated in por-Si at room temperature at $p=760$ Torr [16]. On the one hand, the estimated lifetime of ${ }^{1} \mathrm{O}_{2}$ is significantly shorter than the radiative lifetime of ${ }^{1} \mathrm{O}_{2}$ in at $p=760$ Torr. On the other hand, $\tau_{\Delta} \approx 15 \mathrm{~ms}$ is larger than the 


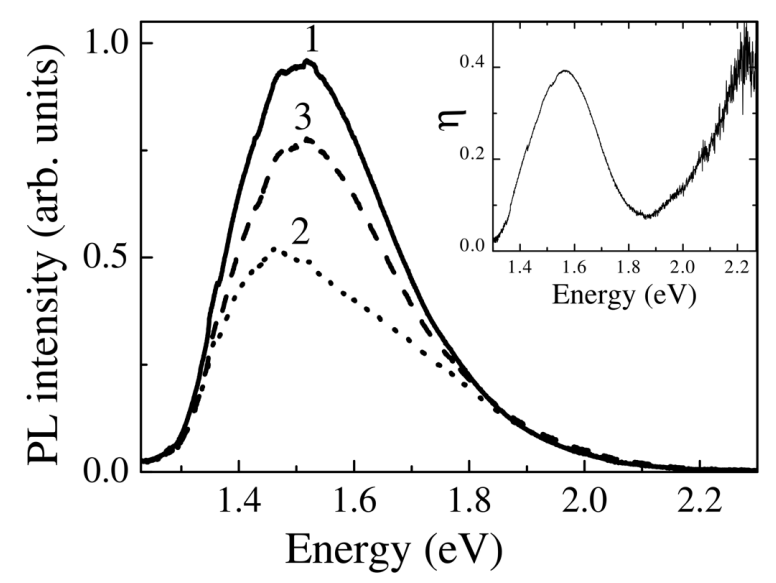

Fig. 11. Spectra of the room-temperature PL of por-Si in water without (1) and with dissolved oxygen at $p=760$ Torr (2) and just after the $\mathrm{O}_{2}$ degassing (3). Inset shows the spectral dependence of the efficiency of energy transfer

lifetime of ${ }^{1} \mathrm{O}_{2}$ in water $(3 \mu \mathrm{s})$ or in biological systems $(<1 \mu \mathrm{s})[9-12]$. This fact implies that por-Si can be considered as the ${ }^{1} \mathrm{O}_{2}$ photosensitizer in biosystems.

\subsection{Photosensitization in liquids}

The standard method to detect ${ }^{1} \mathrm{O}_{2}$ in solutions is to use biochemical traps (scavengers) and to analyze the specific reactions induced by ${ }^{1} \mathrm{O}_{2}$. Typical biochemical traps are cholesterol, 1,3-diphenylisobenzofuran (DPBF), sodium azide, etc. [1, 4]. DPBF was employed as a scavenger of ${ }^{1} \mathrm{O}_{2}$ photosensitized by por-Si [13]. The obtained results demonstrated the ${ }^{1} \mathrm{O}_{2}$ formation via the photosensitization by por-Si. The photoluminescence (phosphorescence) at $0.98 \mathrm{eV}$ from ${ }^{1} \mathrm{O}_{2}$ generated by the energy transfer from por-Si was observed at room temperature in $\mathrm{C}_{6} \mathrm{~F}_{6}$ solution $\left(\tau_{\Delta}=25 \mathrm{~ms}\right)$ [20]. Besides the broad PL band of excitons at $1.6 \mathrm{eV}$, the PL spectrum of a por-Si powder in a $\mathrm{C}_{6} \mathrm{~F}_{6}$ solution after the saturation with oxygen consisted of the ${ }^{1} \mathrm{O}_{2}$ $\left({ }^{1} \Delta\right)$ emission line. The latter was not detected when the benzene $\left(\mathrm{C}_{6} \mathrm{H}_{6}\right)$ instead of $\mathrm{C}_{6} \mathrm{~F}_{6}$ was used as a solution, also the intensity of the exciton PL was nearly the same. The very efficient nonradiative relaxation of the ${ }^{1} \Delta$ state via the energy transfer to the $\mathrm{C}-\mathrm{H}$ vibration of benzene is responsible for the disappearance of the ${ }^{1} \mathrm{O}_{2}$ emission line. The photosensitized generation of ${ }^{1} \mathrm{O}_{2}$ in $\mathrm{C}_{6} \mathrm{~F}_{6}$ was observable under the excitation by light with a broad range of photon energies [20]. This spectral range, which is obviously determined by the absorption coefficient of por-Si, evidences the indirect excitation of ${ }^{1} \mathrm{O}_{2}$ by the energy transfer from nc-Si. The broad excitation spectrum of the ${ }^{1} \mathrm{O}_{2}$ luminescence is apparently differ-

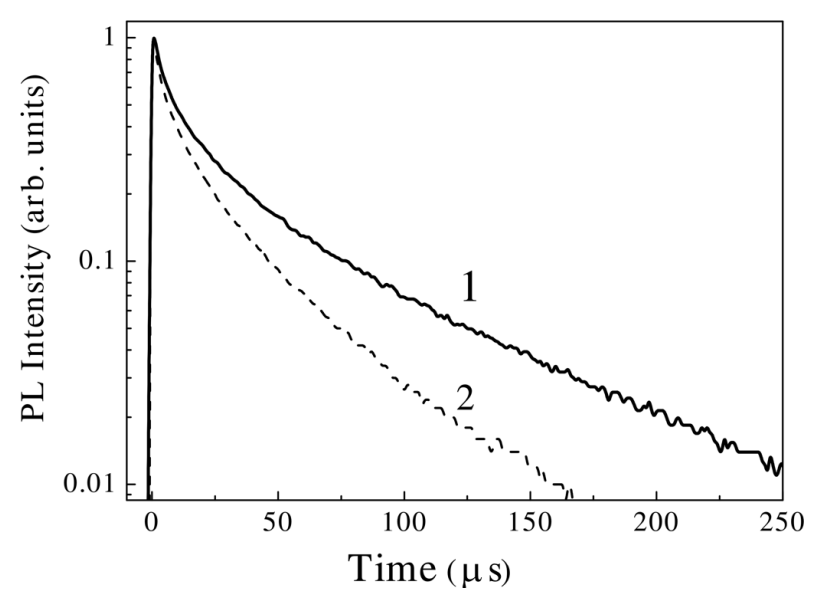

Fig. 12. Transients of the room-temperature PL at $1.63 \mathrm{eV}$ of por$\mathrm{Si}$ in water without (1) and with dissolved oxygen at $p=760$ Torr (2)

ent from that obtained for $\mathrm{C}_{6} \mathrm{~F}_{6}$ not containing por-Si. In this case, ${ }^{1} \mathrm{O}_{2}$ is generated under the excitation at the photon energy, which is resonant to the ${ }^{3} \Sigma \rightarrow{ }^{1} \Sigma$ transition energy in $\mathrm{O}_{2}$.

For biomedical applications of singlet oxygen, its generation in oxygen-containing aqueous solutions is required. Unfortunately, the fast nonradiative relaxation of ${ }^{1} \mathrm{O}_{2}$ in water (see Section 2) makes practically impossible to detect the ${ }^{1} \mathrm{O}_{2}$ luminescence in aqueous solutions. In this case, the ${ }^{1} \mathrm{O}_{2}$ photosensitization can be indirectly probed by monitoring the intensity and the lifetime of the exciton PL of por-Si. Figure 11 shows the typical PL spectra of a por-Si powder dispersed in oxygen-saturated water and in water after the $\mathrm{O}_{2}$ degassing. Similarly to the gaseous ambient (see Fig. 5), $\mathrm{O}_{2}$ dissolved in water causes the spectrally selective PL quenching (inset in Fig. 11). Although the PL quenching strength in water is weaker, its spectral shape is similar to that measured in gaseous $\mathrm{O}_{2}$ ambient. Note that the relative contribution of the high-energy quenching at $2-2.2 \mathrm{eV}$ is stronger for por-Si in water than in the case of gaseous ambient (compare insets in Figs. 5 and 11). This implies that the nc-Si charging or/and $\mathrm{O}_{2}^{-}$formation is more efficient in the presence of polar molecules of water, which can decrease barriers for the charge trapping on the nc-Si surface.

The lifetime of the exciton PL of por-Si dispersed in water was found to decrease after the saturation of the solution by $\mathrm{O}_{2}$ (see Fig. 12). The energy transfer times can be determined from transients of the exciton PL following the procedure described by Eq. (3). The spectral dependence of the energy transfer time is similar to that observed for por-Si in gaseous oxygen ambient. A 
slightly longer energy transfer time correlates with the weaker quenching of the PL intensity of por-Si in water than that in gaseous $\mathrm{O}_{2}$. This fact indicates an influence of water molecules on the exciton recombination and/or energy transfer from excitons to $\mathrm{O}_{2}$ molecules. Indeed, the energy transfer is mostly efficient for nc-Si with hydrogenated (H-terminated) surfaces, which are hydrophobic [32]. Therefore, por-Si dispersed in water should be partially oxidized, which results in decreasing the ${ }^{1} \mathrm{O}_{2}$ photosensitization efficiency [6].

The oxidation of nc-Si in por-Si dispersed in water or aqueous solutions seems to be the main limiting factor to achieve the stable and efficient photosensitization of the $\mathrm{O}_{2}$ generation. The oxidation process can be also enhanced by ${ }^{1} \mathrm{O}_{2}$ photosensitized by por-Si [16, 43]. In order to overcome this serious limitation for the application of por-Si as an efficient photosensitizer of ${ }^{1} \mathrm{O}_{2}$, special procedures of controllable oxidation and/or surface modification of nc-Si are probably required. This can be done, for example, by using the rapid thermal annealing or laser irradiation. In fact, nearly spherical colloidal nc-Si with a mean size of $2.4 \mathrm{~nm}$ obtained by the femtosecond laser ablation in heavy water was found to be an efficient photosensitizer of the ${ }^{1} \mathrm{O}_{2}$ generation in water. The ${ }^{1} \mathrm{O}_{2}$ light emission at $1270 \mathrm{~nm}$ was observed in a colloidal solution of ablated nc-Si [45]. The measured lifetime of about $28 \mu \mathrm{s}$ is only twice shorter than $\tau_{\Delta}$ in pure $\mathrm{D}_{2} \mathrm{O}$. This means that the quenching of ${ }^{1} \mathrm{O}_{2}$ was not very efficient in the prepared solution. Moreover, nc-Si subjected to a $532-\mathrm{nm}$ pulsed laser irradiation for $1 \mathrm{~h}$ at $200 \mathrm{~mW} / \mathrm{cm}^{2}$ did not show signs of the photobleaching of the ${ }^{1} \mathrm{O}_{2}$ generation, providing a decisive advantage over conventional organic photosensitizers [45].

\section{Biomedical Applications of the Singlet Oxygen Formation by Por-Si}

The above-discussed efficient photosensitization of ${ }^{1} \mathrm{O}_{2}$ by nc-Si in various media stimulates experiments on the biomedical verification of the expected photodynamic action. It was found that a photoexcited por-Si powder could suppress the proliferation (permanent division) of cancer cells [23, 46]. For these biomedical experiments, nc-Si was prepared by using the conventional method of electrochemical etching of c-Si wafers in hydrofluoric acid solutions [7, 8]. The obtained free-standing por-Si films were dried in air and then milled to get a powder with the maximal grain size of the order of 10 microns. According to the Raman scattering data, the mean sizes of nc-Si in the initial por-Si layers and in the powder formed from them were about 3-4 nm. Then the por-Si powder was dispersed in pure water bubbled with oxygen to get a homogeneous aqueous suspension. The dry powder was investigated in vacuum and in gaseous oxygen ambient under the excitation by a nitrogen-laser radiation at a wavelength of $337 \mathrm{~nm}$. It was found that the exciton PL intensity of nc-Si in oxygen-saturated water decreases by a factor of $1.5-2$ in comparison with that in oxygen-free water (or in vacuum).

Additional information on the ${ }^{1} \mathrm{O}_{2}$ photosensitization efficiency was obtained by analyzing the PL transients at $1.63 \mathrm{eV}$ of nc-Si excited by laser pulses. The relaxation time of the exponential part of the PL transients in a solution without $\mathrm{O}_{2}$ was $\tau_{\mathrm{ex}} \approx 85 \mu \mathrm{s}$, which is close to the natural radiative lifetime of excitons confined in nc-Si (see Section 2). The saturation of the solution with gaseous $\mathrm{O}_{2}$ at a pressure of 760 Torr leads to a decrease in the PL dacay time to $\tau_{\mathrm{D}} \approx 50 \mu \mathrm{s}$. The ratio $\tau_{\mathrm{ex}} / \tau_{\mathrm{D}} \approx 1.6$ is close to the measured PL quenching. These observations indicate that the decrease of the PL lifetime of nc-Si in oxygen-saturated suspensions was caused by the photosensitized generation of ${ }^{1} \mathrm{O}_{2}$.

The biomedical experiments in vitro were carried out by using cancer cells of 3T3 NIH (mouse fibroblast) grown by the standard procedure of their sub cultivation in the Dulbecco-modified Eagle's medium using 96-well plates or dishes. The cells were cultivated during 1.5 days, and then the medium was replaced by a fresh nutrient solution with various concentrations of nc-Si. One part of the wells was illuminated by a mercury lamp, another part was kept in darkness, and some cells were used for control (without nc-Si and illumination). The inhibitory or lethal effect of the illumination was obtained by measuring the cell quantity in the non-illuminated and illuminated wells per cells quantity in the control wells in which the nc-Si suspension was not added. For this purpose, the method of cells staining by crystal violet was used. Additionally, the cell cycle analysis was performed by using a flow cytofluorimeter [46].

Dependence of the cancer cell number (relative to the control) as a function of the concentration of a por-Si powder in the solution is given in Fig. 13. The number of survived cells after the irradiation in the growth medium with a por-Si concentration of about $0.5 \mathrm{~g} / 1$ decreases strongly as compared to that in the reference group. The death of $80 \%$ of cells was detected for the concentration higher $2 \mathrm{~g} / \mathrm{l}$. At the same time, the effect of por-Si was almost absent over the entire concentration range in darkness. This fact is concerned with the ${ }^{1} \mathrm{O}_{2}$ generation under the illumination in the active solution with dispersed nc-Si, because ${ }^{1} \mathrm{O}_{2}$ being a strong oxidiz- 


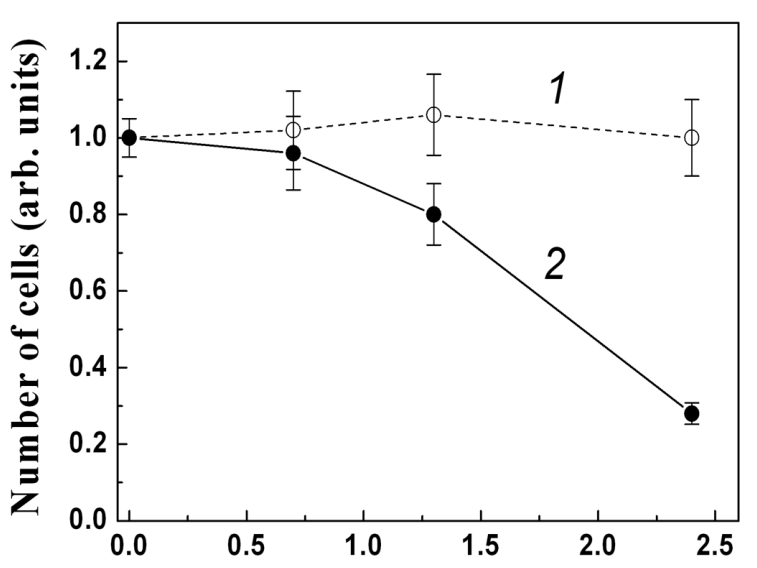

Concentration of nc-Si (g/l)

Fig. 13. Number of cancer cells in darkness (1) and after illumination (2) vs the nc-Si concentration in nutrient solution [23]

ing substance results in both the killing of cells and the inhibition of cell proliferation.

Figure 14 shows typical histograms of the DNA content for mouse fibroblasts kept in a nutrient solution with dispersed nc-Si $(1.5 \mathrm{~g} / \mathrm{l})$. It should be noted that cells with the smallest DNA content (typically less than 100) can be considered as apoptotic cells (dead due to the apoptosis mechanism, i.e. programmed cell death [47]. One can see from Fig. 14 that the illumination of the suspension of cells with added nc-Si leads to an increase of the distribution peak at low DNA contents, which demonstrates an enhancement of the apoptosis rate because of the ${ }^{1} \mathrm{O}_{2}$ generation.

The detected death of cancer cells occurred due likely to the action of photosensitized active oxygen, in particular, to the oxidation of the cell substance by ${ }^{1} \mathrm{O}_{2}$. In addition, the effect of other active forms of oxygen is also possible, e.g., superoxide $\left(\mathrm{O}_{2}^{-}\right.$ions) whose formation is also possible. To reveal the particular mechanisms of the interaction between photoexcited nc-Si and biological objects, additional investigations are still required.

\section{Conclusions}

The experimental studies of microporous Si composed from Si nanocrystals with sizes of several nanometers revealed their remarkable properties to photosensitize active molecular oxygen species such as singlet oxygen. The photosensitization of the singlet oxygen generation by por-Si was found to occur because of the energy transfer from photoexcited Si nanocrystals to oxygen

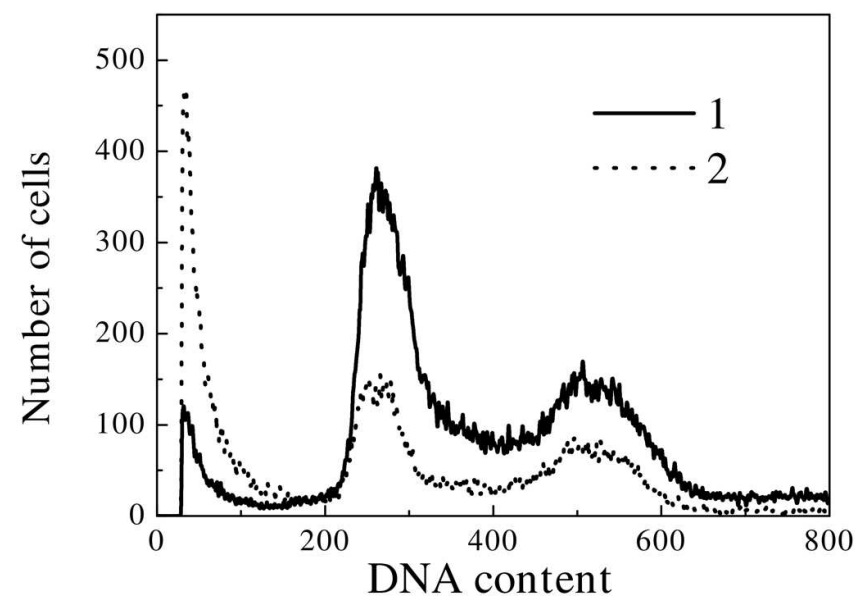

Fig. 14. Typical histograms of DNA for the cancer cells kept in the nutrient solution with dispersed nc-Si (1.5 g/l) in darkness (1) and after illumination (2) [46]

molecules adsorbed on the nanocrystal surfaces. The singlet oxygen generation was observed for por-Si in various gaseous and liquid ambiences. The effect of photoexcited Si nanocrystals on the cancer cell proliferation was observed in vitro. While these observations open a way for in vivo experiments and then to possible applications of por-Si in PDT of malignant tumors, the real progress in this direction is still insignificant.

Main problems for the extended biomedical application of por-Si as a singlet oxygen photosensitizer concern the preparation of stable water-soluble por-Si-based materials with a high efficiency of the singlet oxygen generation. The recently observed biocompatibility and biodegradability of por-Si [48, 49] have to be combined with the photosensitization efficiency and modern biomedical requirements for PDT [50]. A good indication for the ability of nc-Si prepared by the laser ablation to photosensitize the efficient singlet oxygen generation was recently revealed [45]. It seems that the recent progress in formation methods of nc-Si will result in new interesting results of their biomedical applications.

The author is grateful to Dr. L.A. Osminkina, Dr. A.A. Kudryavtsev, Dr. Yu.V. Ryabchikov, and Dr. I.A. Belogorokhov for their assistance in the experimental studies presented in this paper, as well as to Prof. P.K. Kashkarov and Prof. D.I. Kovalev for the helpful discussions and the inestimable contribution to this work.

1. S.J. Arnold, M. Kubo, and E.A. Ogryzlo, Advan. Chem. Ser. 77, 133 (1968). 
2. Photodynamic Tumor Therapy: 2nd and 3rd Generation Photosensitizers, edited by J.G. Moser (Harwood, Amsterdam, 1998).

3. N.J. Turro, Modern Molecular Photochemistry (Univ. Sci. Books, Mill Valley, CA, 1991).

4. D.R. Kearns, Chem. Rev. 71, 395 (1971).

5. D. Kovalev, E. Gross, N. Künzner, F. Koch, V.Yu. Timoshenko, and M. Fujii, Phys. Rev. Lett. 89, 137401 (2002).

6. E. Gross, D. Kovalev, N. Künzner, F. Koch, V.Yu. Timoshenko, and M. Fujii, Phys. Rev. B 68, 115405 (2003).

7. O. Bisi, S. Ossicini, and L. Pavesi, Surf. Sci. Rep. 38, 1 (2000).

8. D. Kovalev, H. Heckler, G. Polisski, and F. Koch, Phys. Stat. Sol. (b) 215, 871 (1999).

9. A.A. Krasnovsky, jr., S.Yu. Egorov, O.V. Nasarova, B.I. Yartsev, and G.V. Ponamarev, Studia Biophys. 124, 123 (1988).

10. A.A. Krasnovsky, jr., Membr. Cell Biol. 12(5), 665 (1998)

11. A.A. Krasnovsky, jr., N.N. Drozdova, Ya.V. Roumbal, A.V. Ivanov, abd R.V. Ambartzumian, Chinese Optics Lett. 3, S1 (2005).

12. A.A. Krasnovsky, jr., Ya.V. Roumbal, A.V. Ivanov, and R.V. Ambartzumian, Chem. Phys. Lett. 430, 260 (2006).

13. M. Fujii, M. Usui, Sh. Hayashi, E. Gross, D. Kovalev, N. Künzner, J. Diener, and V.Yu. Timoshenko, J. Appl. Phys. 95, 3689 (2004).

14. D. Kovalev and M. Fujii, Adv. Mat. 17, 2531 (2005).

15. V. Chirvony, V. Bolotin, E. Matveeva, V. Parkhutik, J. Photochem. Photobiol. A 181, 106 (2006).

16. M.B. Gongalsky, E.A. Konstantinova, L.A. Osminkina, and V.Yu. Timoshenko, Semicond. 44, 89 (2010).

17. E.A. Konstantinova, V.A. Demin, A.S. Vorontzov, Yu.V. Ryabchikov, I.A. Belogorokhov, L.A. Osminkina, P.A. Forsh, P.K. Kashkarov, and V.Yu. Timoshenko, J. Non-Cryst. Solids 352, 1156 (2006).

18. E.A. Konstantinova, V.A. Demin, V.Yu. Timoshenko, and P.K. Kashkarov, JETP Lett. 85, 59 (2007).

19. E.A. Konstantinova, V.A. Demin, and V.Yu. Timoshenko, J. Exp. Theor. Phys. 107, 473 (2008).

20. M. Fujii, S. Minobe, M. Usui, Sh. Hayashi, E. Gross, J. Diener, and D. Kovalev, Phys. Rev. B 70, 085311 (2004).

21. M. Fujii, M. Usui, Sh. Hayashi, E. Gross, D. Kovalev, N. Künzner, J. Diener, and V.Yu. Timoshenko, Phys. Stat. Sol. (a) 202, 1385 (2005).

22. M. Fujii, D. Kovalev, B. Goller, S. Minobe, Sh. Hayashi, and V.Yu. Timoshenko, Phys. Rev. B 72, 165321 (2005).

23. V.Yu. Timoshenko, A.A. Kudryavtsev, L.A. Osminkina, A.S. Vorontzov, Ya.V. Ryabchikov, I.A. Belogorokhov,
D. Kovalev, and P.K. Kashkarov, JETP Lett. 83, 423 (2006).

24. H.H. Wasserman, Introductory remarks. in Singlet Oxygen, edited by H.H. Wasserman (Academic Press, New York, 1979).

25. H. Kautsky, Trans. Faraday Soc. 35, 216 (1939).

26. T. Förster, Ann. der Phys. 2, 55 (1948).

27. D.L. Dexter, J. Chem. Phys. 21, 836 (1953).

28. S.Yu. Egorov, V.F. Kamalov, N.I. Koroteev, A.A. Krasnovsky, jr., B.N. Toleutaev, and S.V. Zinukov, Chem. Phys. Lett. 163, 421 (1989).

29. R. Schmidt and H.-D. Brauer, J. Am. Chem Soc. 109, 6976 (1987).

30. J.W. Snyder, E. Skovsen, J.D.C. Lambert, and P.R. Ogilby, J. Am. Chem. Soc. 127, 14558 (2005).

31. A. Uhlir, Bell Syst. Tech. 35, 333 (1956).

32. A.G. Cullis, L.T. Canham, and P.D.J. Calcott, J. Appl. Phys. 82, 965 (1997).

33. G.D. Sanders and Y.-C. Chang, Phys. Rev. B 45, 9202 (1992).

34. B. Delley and E.F. Steigmeier, Appl. Phys. Lett. 67, 2370 (1995).

35. G.C. John and V.A. Singh, Phys. Rep. 263, 93 (1995).

36. D.A.G. Bruggeman, Ann. der Phys. 24, 636 (1935).

37. P.D.J. Calcott, K.J. Nash, L.T. Canham, M.J. Kane, and D. Brumhead, J. Phys., Condens. Matter 5, L91 (1993).

38. D.G. Yarkin, E.A. Konstantinova, and V.Yu. Timoshenko, Semicond. 29, 348 (1995).

39. I.V. Blonsky, M.S. Brodin, V.A. Thoryk, A.G. Filin, and Ju.P. Piryatinskij, Semicond. Sci. Technol. 12, 11 (1997).

40. A.N. Obraztsov, V.Yu. Timoshenko, H. Okushi, and H. Watanabe, Semiconductors 31, 534 (1997).

41. Yu.V. Ryabchikov, I.A. Belogorokhov, A.S. Vorontsov, L.A. Osminkina, V.Yu. Timoshenko, and P.K. Kashkarov, Phys. Stat. Sol. (a) 204, 1271 (2007).

42. Yu.V. Ryabchikov, I.A. Belogorokhov, M.B. Gongalsky, L.A. Osminkina, and V.Yu. Timoshenko, Semicond. 204, 1271 (2011).

43. D. Kovalev, E. Gross, J. Diener, V.Yu. Timoshenko, and M. Fujii, Appl. Phys. Lett. 85, 3590 (2004).

44. I.J. Langmuir, J. Am. Chem. Soc. 38, 2221 (1916).

45. D. Rioux, M. Laferriere, A. Douplik, D. Shah, L. Lilge, A. Kabashin, M.M. Meunier, and J. Biomedic. Optics 14, 021010 (2009). 
46. V.Yu. Timoshenko, L.A. Osminkina, A.S. Vorontzov, Ya.V. Ryabchikov, M.B. Gongalsky, A.I. Efimova, E.A. Konstantinova, T.Yu. Bazylenko, P.K. Kashkarov, and A.A. Kudryavtsev, Proc. of SPIE 60606, 66061E (2007).

47. H. Steller, Science 267, 1445 (1995).

48. L.T. Canham, Nanotechn. 18, 85704 (2007).

49. J.-H. Park, L. Gi, G. von Maltzahn, E. Ruoslahti, S.N. Bhatia, and M. Sailor, Nature Mat. 8, 331 (2009).

50. D.E.J.G.J. Dolmans, D. Fukumura, and R.K. Jain, Nature Rev. Cancer 3, 380 (2003).

Received 30.08.11
ДОСЛІДЖЕННЯ ФОТОЛЮМІНЕСЦЕНЦІЇ ПОРИСТОГО КРЕМНІЮ ЯК ФОТОСЕНСИБІЛІЗАТОРА ГЕНЕРАЦЇ̈ СИНГЛЕТНОГО КИСНЮ

В.Ю. Тимошенко

Р ез ю м е

Нанокристали кремнію із розмірами кілька нанометрів в шаpax та порошках пористого кремнію виявляють якості фотосенсибілізаторів генерації синглетного кисню, що є хімічно дуже активною формою молекулярного кисню. Механізм та ефективність фотосенсибілізації вивчено за допомогою фотолюмінесцентної спектроскопії. Експериментальні факти проаналізовано у світлі майбутніх біомедичних застосувань пористого кремнію. 